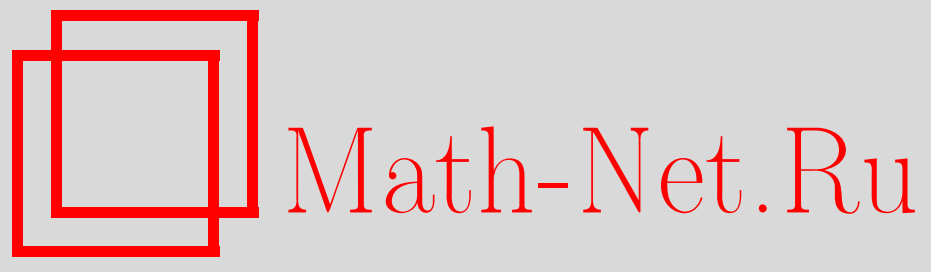

М. И. Штогрин, Специальные изометрические преобразования цилиндра, УМН, 2007, том 62, выпуск 5, 173-174 DOI: https://doi.org/10.4213/rm7599

Использование Общероссийского математического портала Math-Net.Ru подразумевает, что вы прочитали и согласны с пользовательским соглашением http://www . mathnet.ru/rus/agreement

Параметры загрузки:

IP: 34.229 .108 .108

26 апреля 2023 г., 12:18:19

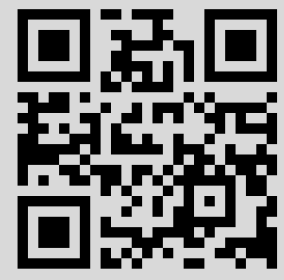




\section{Специальные изометрические преобразования цилиндра}

\section{М. И. Штогрин}

Исследуются специальные изометрические вложения поверхности прямого кругового иилиндра в трехмерное евклидово пространство $\mathbb{R}^{3}$, см. [1]. Строение вложенной цилиндрической поверхности обладает двоякой периодичностью. В окружном направлении действует группа $G=n m m$, порожденная отражениями от плоскостей, угол между которыми равен $\pi / n$. По высоте действует группа параллельных переносов. Периодом последней может служить боковая поверхность призмы.

Возьмем вспомогательную правильную призму П с горизонтальным $2 n$-угольным основанием и вертикальной осъю. На боковой грани $\alpha_{1}$ возьмем произвольную гладкую кривую $\gamma_{1}$, однозначно проектирующуюся на ось. На смежной боковой грани $\alpha_{2}$ построим кривую $\gamma_{2}$, получающуюся из $\gamma_{1}$ при зеркальном отражении от плоскости $\beta$, проходящей через боковое ребро $\alpha_{2} \cap \alpha_{1}$ и ось. Аналогично строим кривую $\gamma_{3}$ на грани $\alpha_{3}$, смежной с $\alpha_{2}$, и т. д. Так на каждой грани $\alpha_{i}$ получим кривую $\gamma_{i}$. Проведем теперь через кривые $\gamma_{1}$ и $\gamma_{2}$ цилиндрическую поверхность $Z_{12}$ с горизонтальными образующими, перпендикулярными плоскости $\beta$. Аналогично строим цилиндрические поверхности $Z_{23}, Z_{34}$ и т. д. Все вместе они составляют кусочно гладкую трубчатую поверхность $Z$ с ребристыми линиями $\gamma_{i}, i=1, \ldots, 2 n$.

Именно так в работе [1] построил А.В. Погорелов открытую им трубчатую поверхность $Z$. Она всегда изометрична прямому круговому цилиндру, см. [1; с. 51]. Постоянный периметр $L$ всех горизонтальных сечений поверхности $Z$ связан с радиусом $r$ цилиндра соотношением $L=2 \pi r$. Высота цилиндра равна длине кривой $\widetilde{\gamma}=\beta \cap Z_{12}$. Предположив, что кривая $\gamma_{1}$ на грани $\alpha_{1}$ призмы П, с помощью которой была получена поверхность $Z$, произвольным образом деформируется, но так, что длина кривой $\widetilde{\gamma}=\beta \cap Z_{12}$ сохраняется, А. В. Погорелов получил такую деформацию кусочно гладкой поверхности $Z$, которая является геометрическим изгибанием, см. [1; с. 52]. (Этот важный факт об изометрических вложениях цилиндра следовало осветить в работе [2] в явном виде. В замечании 1 нужно было четко и ясно сказать, что в процессе изгибания поверхности $T$ высота всех трапеций сохраняется.)

Если при изгибании в точках $\gamma_{i}$ непрерывно уменьшать кривизну кривой, устремив ее к нулю, то кривая $\gamma_{i}$ выпрямится в прямолинейный отрезок $\ell_{i}$, а кусочно гладкая поверхность $Z$ превратится в изометричную кусочно линейную поверхность $\bar{Z}$.

$\mathrm{K}$ краям кусочно линейной поверхности $\bar{Z}$ приклеим два выпуклых многоугольника. Получим выпуклый многогранник. Тут $\ell_{j} \cap \ell_{i}=\varnothing, j \neq i$. В любой вершине сходятся три плоских угла $\varphi, \pi-\varphi$ и $\pi-\pi / n$. В силу неравенств треугольника имеем

$$
\frac{\pi}{2 n}<\varphi<\pi-\frac{\pi}{2 n}
$$

Этим же неравенствам удовлетворяет и переменный угол, который составляет кривая $\gamma_{i}$ в любой своей точке с той или иной прямолинейной образующей, проходящей через данную точку в случае произвольной кусочно гладкой поверхности $Z$.

Разрежем цилиндр $Z_{2 n 1}$ плоскостью симметрии, т. е. одной из $n$ плоскостей симметрии поверхности $Z \subset \mathbb{R}^{3}$, и развернем поверхность $Z$ на евклидову плоскость $\mathbb{R}^{2}$. Получим развертку кусочно гладкой поверхности $Z$ в виде некоторого прямоугольника $R \subset \mathbb{R}^{2}$, обладающего парой эквивалентных сторон. Обе стороны имеют один и тот же образ на поверхности $Z$ при изометрическом отображении $f: R \rightarrow Z$. Так как отображение $f$ сохраняет углы, то развертка $R$ наследует условия $(*)$.

Работа выполнена при финансовой поддержке РФФИ (грант № 05-01-00170), программы "Ведущие научные школы" (проект НШ-4182.2006.1) и государственной программы фундаментальных исследований ОМН РАН "Современные проблемы теоретической математики”. 
Пусть развертка $R$ кусочно гладкой поверхности $Z$ расположена на плоскости $\mathbb{R}^{2}$ так, что прообразы всех прямолинейных образующих поверхности $Z$ находятся на горизонтальных прямых. Тогда неравенства $(*)$ означают, что график прообраза гладкой кривой $\gamma_{i}$ на плоскости $\mathbb{R}^{2}$ является не слишком пологим. Прообразы следов плоскостей зеркальной симметрии на трубчатой поверхности $Z$ суть вертикальные отрезки. Они разбивают развертку $R$ на $2 n$ фундаментальных прямоугольников прообразов фундаментальных областей группы $G=n m m$. В каждом прямоугольнике имеется прообраз своей гладкой кривой $\gamma_{i}$, взятой на грани $\alpha_{i}$ призмы П.

Теорема. Любая не слишком пологая (см. (*)) гладкая кривая $\tau_{1}$, расположенная на развертке $R$ внутри заданного фундаментального прямоугольника, является прообразом некоторой гладкой кривой $\gamma_{1}$, взятой на грани $\alpha_{1}$ призмы П и порождающей кусочно гладкую поверхность $Z$, построенную методом А. В. Погорелова.

Пусть $\bar{Z}$ и $Z$ изометричны. Тогда их развертки можно наложить друг на друга.

СледСтвиЕ. Не слишком пологая вариация прообраза ребра $\ell_{1}$ на развертке $R$ влечет вариачию гладкой кривой $\gamma_{1}$, задающую изгибание поверхности $Z$.

Сказанное выше является всего лишь иным изложением работы $[1 ; \S 3$, п. 1].

А. В. Погорелов рассматривал лишь общий случай кусочно гладких преобразований цилиндра. Теперь мы рассматриваем и некоторые частные случаи. Среди них встречаются кусочно гладкие, кусочно линейные и экзотические поверхности.

Особо выделим один частный случай, даже серию. В качестве исходной кривой $\gamma_{1}$ возьмем гладкую кривую, соединяющую вершину нижнего основания с серединой верхнего основания грани $\alpha_{1}$ вспомогательной правильной призмы П. Кривая $\gamma_{1}$ однозначно проектируется на ось и вдобавок обладает вертикалъными касательными в обоих концах. Так как призма П имеет $2 n$-угольное основание, то нижнее основание поверхности $Z$ есть правильный $n$-угольник, а верхнее - правильный $2 n$-угольник.

Именно так построим $N$ поверхностей $Z_{k}, k=1, \ldots, N$. При этом в качестве основания соответствующей вспомогательной призмы $\Pi_{k}$ берем правильный $2^{k} n$-угольник. Пусть высота всех призм $\Pi_{k}$ равна 1 , и пусть нижнее основание поверхности $Z_{k}$, $k=2, \ldots, N$, совпадает с верхним основанием поверхности $Z_{k-1}$. Тогда $\bigcup_{k=1}^{N} Z_{k}$ представляет собой конечную кусочно гладкую трубчатую поверхность, изометричную цилиндру. При $N \geqslant 2$ поверхность $\bigcup_{k=1}^{N} Z_{k}$ состоит из $2^{N} n>2 n$ гладких кусков. Размножив поверхность $\bigcup_{k=1}^{N} Z_{k}$ всеми элементами группы $H$, порожденной отражениями $m_{1}$ и $m_{2}$ от плоскостей нижнего и верхнего основания соответственно, получим бесконечную в обе стороны кусочно гладкую поверхность, обладающую двоякой периодичностью. В окружном направлении действует группа $G=n m m$ порядка $2 n$. Однако полученная бесконечная поверхность содержит не $2 n$, а неограниченное множество гладких кусков, что противоречит выводу, сделанному в [1; с. 53].

Уточнение. Данный вывод выполняется, если $\gamma_{j} \cap \gamma_{i}=\varnothing$ при всех $j \neq i$.

В нашем частном случае при некоторых $j \neq i$ имеет место неравенство $\gamma_{j} \cap \gamma_{i} \neq \varnothing$. Все сказанное является существенным дополнением к работе А. В. Погорелова [1].

\section{Список литературы}

[1] А. В. Погорелов, Изгибания поверхностей и устойчивость оболочек, Наука, М., 1986; 1998. [2] М. И. Штогрин, УМН, 60:4 (2005), 221-222.

М. И. Штогрин (М. I. Shtogrin)

Математический институт им. В. А. Стеклова РАН

E-mail: stogrin@mi.ras.ru
Представлено В. М. Бухштабером Принято редколлегией 01.08.2007 